\title{
Effects of bilateral orbital decompression by an endoscopic endonasal approach in dysthyroid orbitopathy
}

\author{
A Neugebauer, K Nishino, P Neugebauer, W Konen, O Michel
}

\begin{abstract}
Aims-The efficacy of endoscopic endonasal orbital decompression in dysthyroid orbitopathy was analysed.

Methods-In 21 consecutive cases of bilateral operation short term (10 (SD 6) days after operation) and long term (156 (12) days after operation) results were recorded.
\end{abstract}

Results-Short term results showed that vision of the more affected eye improved from a mean of 0.35 to 0.59 ; vision improved in all but one eye which remained unchanged. In the fellow eyes mean visual acuity improved from 0.6 to 0.7 ; three of these eyes showed a decrease. Mean proptosis returned from $23.0 \mathrm{~mm}$ to $20.0 \mathrm{~mm}$. As to motility the mean abductive capacity decreased from $5.5 \mathrm{~mm}$ to $4.0 \mathrm{~mm}$ of monocular excursion, whereas adduction increased from $7.5 \mathrm{~mm}$ to $8.5 \mathrm{~mm}$. Upgaze and downgaze did not show any major change. The mean angle of horizontal squint shifted from $7.5^{\circ}$ of convergence to $15 \cdot 5^{\circ}$ while no significant vertical or cyclorotational deviation was induced. These immediate postoperative results proved to be stable for the period of long term follow up with only slight changes. No significant bleeding or specific otorhinolaryngological complication without resolve occurred intraoperatively or perioperatively.

Conclusion-This method is believed to be superior to non-endoscopic techniques because it avoids external scars and antral pain. With regard to the relief of intraorbital pressure, the technique gives good results for visual acuity improvement, but in proptosis reduction the method is not as efficient as external or combined procedures. There seems to be no difference when compared with other approaches in induction of horizontal squint. The method has a protective long term effect against the recurrence of compressive optic neuropathy.

(Brf Ophthalmol 1996; 80: 58-62)

In some patients with dysthyroid orbitopathy conservative treatment by immunosuppression or radiotherapy may fail and acute malignant orbitopathy may develop. In these cases vision is threatened by compressive optic neuropathy or by exposure keratopathy and immediate surgical intervention is required in order to relieve intraorbital pressure. First efforts to achieve this stem from the early 20 th century when Dollinger chose an approach to the orbit of a lateral orbitotomy. ${ }^{1}$ Since then other authors have described various techniques used successfully to decompress the orbit. The medial and lateral wall as well as the orbital roof and floor were removed separately or in various combinations. ${ }^{2}$

A combination of the medial and inferior wall removal with longitudinal incision of the periorbita as described in 1957 by Walsh and Ogura ${ }^{3}$ became the standard procedure and is used most often. Approach to the orbital floor may be attained transantrally or transconjunctivally whereas the medial wall is reached by external cutaneous incision or again transconjunctivally.

An endoscopic endonasal approach offers the possibility of medial and inferior wall decompression without the risk of disfiguring external scars and avoiding the typical Caldwell-Luc complications such as scarring and pain in the antral region. In a 1990 study, Kennedy ${ }^{4}$ used this endonasal modification to the Walsh-Ogura procedure but combined it with a lateral orbitotomy in five of the eight patients presented.

Since 1989 we have performed endoscopic endonasal medial and inferior orbital wall decompression as a single technique. We report the results of 21 consecutive bilateral operations.

\section{Subjects and methods}

From October 1989 to October 1994 we performed bilateral orbital decompression in 21 patients. All patients showed signs of raised intraorbital pressure such as compressive optic neuropathy indicated by decreased visual acuity, visual field scotomas, impaired colour vision, or visual evoked cortical potential alteration. In none of the patients was the decision to decompress the orbit taken for cosmetic reasons. In all patients a preoperative computed tomography was performed in order to confirm diagnosis and indication, to exclude a sinusitis which would hinder surgical intervention, and to delineate the individual anatomical configuration of the orbit and paranasal sinuses.

All operations were performed by the same surgeon (OM) according to a standardised technique.

In order to establish wide symmetric nasal cavities a conventional septoplasty was performed where necessary. An endonasal ethmoidectomy with resection of the middle 
turbinate was then performed and the medial orbital wall fully exposed without opening the orbit. The sphenoid sinus was opened, close to the midline with resectioning of the frontal wall. The maxillary sinus was then opened by a middle meatal window with a wide opening of the medial maxillary wall, leaving a wide connection between maxillary sinus, ethmoid sinus, and sphenoid sinus. A piece by piece removal of the bony medial and inferior wall was then performed with preservation of the infraorbital nerve. After bony decompression the periorbita was incised and the orbital fat herniated; care was taken not to affect muscular tissue with this incision procedure. A Merocel (Merocel Corp, Mystic, CT, USA) packing soaked with a solution of doxycycline (Vibramycin, Invicta) was applied and removed on the second day after operation.

For our study we retrospectively analysed the ophthalmic data of patients who underwent this surgical procedure.

Furthermore, the otorhinolaryngological reports were checked for perioperative or postoperative complications. Preoperative and postoperative ophthalmic examination included corrected visual acuity; proptosis measurement by Hertel exophthalmometer; monocular excursion measurement according to Kestenbaum's limbus test of motility for adduction, abduction, elevation, and depression of the globe; and measurement of the maximum angle of squint by dissociating procedures such as alternating prism and cover test or dark red glass test with Harms' tangent screen.

For each of the ophthalmic control variables - visual acuity, proptosis ( $\mathrm{mm})$, monocular excursion (mm), and angle of squint $\left({ }^{\circ}\right)$ - the preoperative value was registered if the examination was no longer than 30 days before operation; in case of multiple examinations during this interval the data of the examination closest to the operation were registered. For short term follow up, postoperative data from examinations within 30 days after operation were considered with preference for examinations nearest to day 14 after operation. For long term follow up, data from examinations between day 120 and 180 were allowed for with preference for examinations nearest to day 150 .

In three of the 21 patients no sufficient preoperative or postoperative data could be obtained because the ophthalmic examinations were not carried out in our ophthalmic department. In some other patients not all variables were measured or date of examination did not fulfil the above mentioned criteria. In order to exclude bias and not to intermingle short and long term follow up data, reduced numbers of data comparing with the whole collective data were allowed. Furthermore, in one patient who suffered from amblyopia in one eye from one patient with two sided maculopathy the visual acuity measurements were not considered. Thus, the numbers of eyes and patients mentioned in the results vary.

Data were analysed by the means of descriptive statistics.
For the analysis of short term follow up, the data for both eyes of each patient were separated into two groups in order to exclude statistical errors induced by intraindividual data grouping. In group 1 data of the more affected eyes were collected whereas group 2 contains the data of the less affected eyes. The more affected eye was defined by visual acuity. The eye with lower acuity was assigned to group 1 , and in cases of equality the eye with higher intraocular pressure was put into group 1 .

\section{Results}

The data of 18 patients, 16 females and two males were considered. The mean age was 53 years with a range of 29 to 74 years. Eleven of these patients had undergone one or more series of systemic steroids and retrobulbar radiation, four had received only systemic steroid treatment, and three neither steroids nor radiation. In some patients systemic steroids were administered before operation to reduce orbital compression. In general this medication was suspended on the first day after operation and therefore steroids had not been given to any of the patients on the date of the postoperative examination analysed for this study.

\section{VISUAL ACUITY}

Figure 1 shows the preoperative versus postoperative visual acuity for the short term follow up. In 18 eyes visual acuity improved, in six eyes it remained unchanged, and in three eyes it decreased. Visual acuity did not decrease in any of the eyes with stronger affection. In group 1 mean visual acuity increased from a preoperative mean of 0.35 (SD 0.26 , median 0.3 ) to a postoperative mean of 0.59 (SD 0.2 , median $0 \cdot 6$ ). In group 2 the preoperative mean was 0.6 (SD 0.18 , median 0.6 ) and postoperative mean was $0 \cdot 7$ (SD $0 \cdot 16$, median $0 \cdot 7$ ). For all eyes mean preoperative visual acuity was 0.47 (SD 0.26 ), mean postoperative visual acuity was 0.64 (SD $0 \cdot 19$ ). A maximum increase in visual acuity of 0.7 and a maximum decrease of 0.4 were registered.

In the long term follow up 22 eyes were examined. In these eyes there was only a slight change in visual acuity in the postoperative period from a mean of $0.63(\mathrm{SD} 0 \cdot 19)$ at the

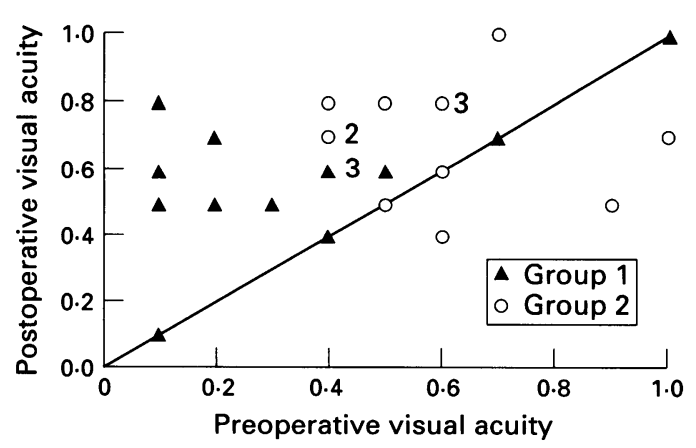

Figure 1 Visual acuity before and 10 days after

decompression. Mean group $1(n=14)$ : preop $0 \cdot 35$ (SD

$0 \cdot 26$, median 0.3 ); postop 0.59 (SD 0.2 , median 0.6 ).

Mean group $2(n=13)$ : preop 0.6 (SD 0.18, median 0.6); postop 0.7 (SD $0 \cdot 16$, median $0 \cdot 7)$. 


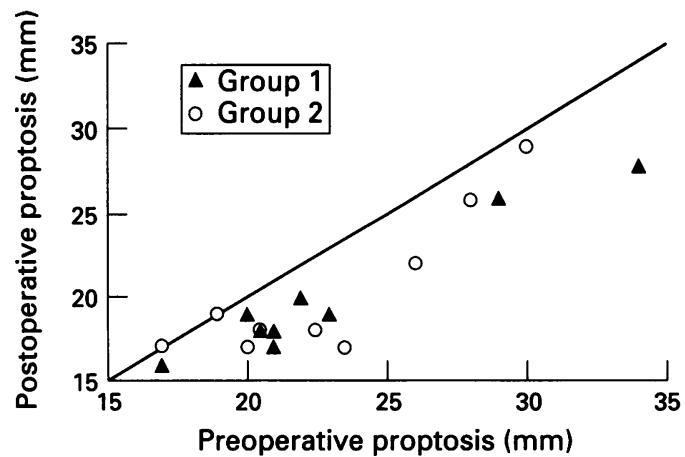

Figure 2 Proptosis before and after decompression. Mean group $1(n=9)$ : preop 23.0 mm (SD 5.0, median 21.0); postop $20.0 \mathrm{~mm}$ (SD 4.0, median 19.0). Mean group 2 $(n=9)$ : preop $23.0 \mathrm{~mm}$ (SD 4.5, median 22.5 ); postop $20 \cdot 5(S D 4 \cdot 5$, median $18 \cdot 0)$.

short term control to a mean of 0.65 (SD 0.24) about 5 months after operation.

\section{PROPTOSIS}

The preoperative versus postoperative proptosis values are indicated in a scatter diagram (Fig 2) for the short term control. Enlarged proptosis did not occur in any of the cases whereas proptosis remained stable in two eyes and receded in 16 eyes. In group 1 proptosis receded from $23.0 \mathrm{~mm}$ (SD $5 \cdot 0$, median $21 \cdot 0$ ) before operation to $20.0 \mathrm{~mm}$ (SD $4 \cdot 0$, median 19.0) after operation. In group 2 a preoperative mean of $23.0 \mathrm{~mm}$ (SD 4.5 , median 22.5 ) was found and a postoperative mean of $20.5 \mathrm{~mm}$ (SD 4.5 , median 18.0). Mean preoperative value of all eyes was $23.0 \mathrm{~mm}$ (SD 4.5 ), mean postoperative value was $20.0 \mathrm{~mm}$ (SD $4 \cdot 0$ ). Maximal regression of proptosis was $6.5 \mathrm{~mm}$.

In eight eyes short term results could be compared with long term results. The mean of $17.5 \mathrm{~mm}$ (SD 2.5 at short term and 1.5 at long term control) after operation did not change.

\section{ANGLE OF SQUINT}

As to the short term results it can be seen from the scatter diagram in Figure 3 that the horizontal squint angle changed to more of a convergent squint in nine patients, while four remained the same, and one showed a slight decrease. Mean angle of horizontal squint was $7.5^{\circ}\left(\mathrm{SD} 7 \cdot 0^{\circ}\right)$ of convergence before and $15 \cdot 5^{\circ}\left(\mathrm{SD} 15 \cdot 0^{\circ}\right)$ of convergence after orbital

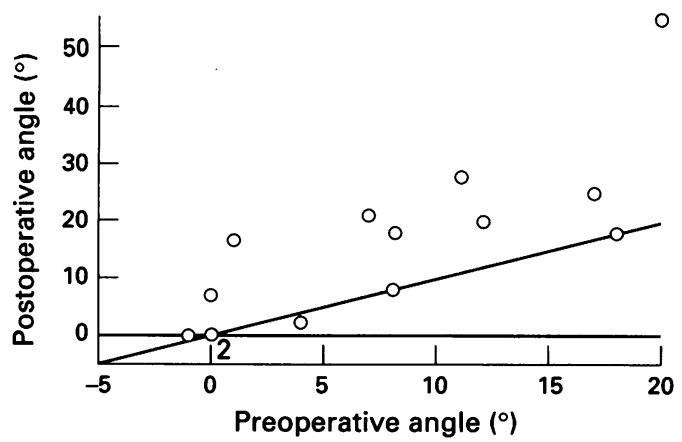

Figure 3 Horizontal squint angle before and 10 days after decompression. Mean $(n=14)$ : preop $7 \cdot 5^{\circ}$ (SD 7.0, median 7.5); postop $15 \cdot 5^{\circ}$ (SD $15 \cdot 0$, median $7 \cdot 5$ ). decompression. In nine patients long term results could be attained, with a shift from $10.0^{\circ}\left(\mathrm{SD} 7 \cdot 5^{\circ}\right)$ of convergence immediately after operation to $13.5^{\circ}\left(\mathrm{SD} 8.0^{\circ}\right)$ after a 5 month period.

Regarding vertical squint, seven patients did not show a deviation preoperatively or postoperatively. Two more patients had no preoperative vertical deviation but developed in the short term follow up a hypertropia of $4.0^{\circ}$ and $1.5^{\circ}$, whereas in one patient a preoperative hypertropia of $7.0^{\circ}$ resolved to $0^{\circ}$ postoperatively. Of the remaining four patients who already showed a preoperative deviation of maximum $6.0^{\circ}$ which postoperatively did not recede to $0^{\circ}$, one improved from $6.0^{\circ}$ to $5.0^{\circ}$ and three changed from left hypertropia of maximum $5.0^{\circ}$ to right hypertropia of maximum $4.0^{\circ}$. In the long term follow up period no major changes occurred with regard to vertical squint.

As to cyclorotatory squint, in nine patients a deviation did not occur preoperatively or postoperatively. In the short term follow up one patient developed an excyclorotation of $5^{\circ}$ while in another patient an excyclorotation of $2^{\circ}$ resolved itself. The remaining three patients showed preoperative excyclorotation of $3^{\circ}$ changing to $4^{\circ}$, of $5^{\circ}$ changing to $4^{\circ}$, and of $4^{\circ}$ changing to an incyclorotation of $9^{\circ}$. Apart from this incyclorotation resolving itself, the long term follow up showed no change when compared with the short term follow up.

\section{ABDUCTION CAPACITY}

Figure 4 shows preoperative versus immediate postoperative abduction capacity. In most cases abduction was reduced, in two eyes it was not affected, and in six eyes it improved. For all eyes mean preoperative abduction capacity was $5.5 \mathrm{~mm}$ (SD $2.0 \mathrm{~mm}$ ), mean postoperative abduction was $4.0 \mathrm{~mm}$ (SD 2.5 $\mathrm{mm})$. Abduction change showed a maximum decrease of $6.0 \mathrm{~mm}$ and a maximum increase of $2.0 \mathrm{~mm}$.

In 16 eyes values for comparison of short term versus long term results were obtained. In these eyes abduction capacity improved slightly from $5.0 \mathrm{~mm}$ to $5.5 \mathrm{~mm}$.

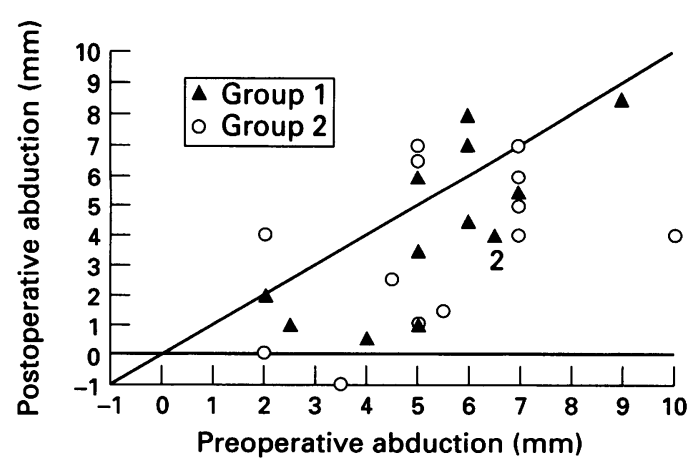

Figure 4 Abduction before and 10 days after decompression. Mean group $1(n=13)$ : preop $5.5 \mathrm{~mm}$ (SD $2 \cdot 0$, median 6.0); postop $4.5 \mathrm{~mm}(S D 2 \cdot 5$, median $4 \cdot 0)$. Mean group $2(n=13)$ : preop $5 \cdot 5 \mathrm{~mm}(S D 2 \cdot 0$, median $5 \cdot 0)$; postop $3.5 \mathrm{~mm}$ (SD $2 \cdot 5$, median $4 \cdot 0)$. 


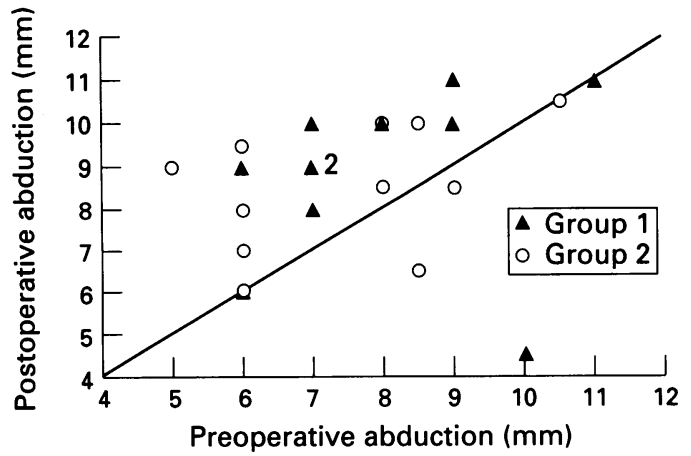

Figure 5 Adduction before and 10 days after decompression. Mean group $1(n=12)$ : preop $8.0 \mathrm{~mm}(S D$ 1.5 , median $7 \cdot 0$ ); postop $9.0 \mathrm{~mm}(S D 2.0$, median 10.0$)$ Mean group $2(n=12)$ : preop $7.5 \mathrm{~mm}(S D) 1 \cdot 5$, median 8.0); postop $8.5 \mathrm{~mm}$ (SD 1.5 , median 9.0$)$

\section{ADDUCTION CAPACITY}

In Figure 5 the preoperative adductive capacity is compared with the values of short term follow up. Postoperatively the majority showed improved adduction, four eyes remained the same, and three eyes showed decreased adduction. Mean adduction was $7.5 \mathrm{~mm}$ (SD 1.5) preoperatively and $8.5 \mathrm{~mm}(\mathrm{SD} 2.0)$ postoperatively. The adduction change showed a maximum decrease of $5.5 \mathrm{~mm}$ and a maximum increase of $4.0 \mathrm{~mm}$.

In 12 eyes we could compare short term and long term follow up data where there was also a slight increase from $9.0 \mathrm{~mm}$ (SD 1.5) to $9 \cdot 5 \mathrm{~mm}$ (SD 1.0).

\section{VERTICAL GAZE MOTILITY}

For all eyes in short term follow up upgaze capacity showed a mean of $3.5 \mathrm{~mm}$ (SD 1.5) preoperatively and a mean of $4.0 \mathrm{~mm}$ (SD 1.5) postoperatively. For downgaze mean preoperative value was $7.5 \mathrm{~mm}(\mathrm{SD} 2.0)$ and postoperative value was $8.0 \mathrm{~mm}$ (SD 2.0). Upgaze in 18 eyes was compared as to short to long term changes. A shift from $4.0 \mathrm{~mm}$ (SD 1.5) to $4.5 \mathrm{~mm}$ (SD 2.0) was registered. Also for downgaze an increase from $7 \cdot 0$ (SD 2.0) to $7 \cdot 5$ (SD 1.5) was found in 14 eyes between the two postoperative examinations.

\section{PERIOPERATIVE AND POSTOPERATIVE ENT FINDINGS}

Intraoperatively no complications occurred. In particular no significant bleeding or damage to the eye muscles when opening the periorbita was observed. Postoperatively two patients complained of mild infraorbital hypaesthesia which receded after some days. Postoperative hospitalisation did not exceed 8 days in any of the cases.

\section{Discussion}

Inferomedial orbital decompression is an acknowledged method to lower intraorbital pressure in cases of dysthyroid orbitopathy with compressive neuropathy of the optic nerve. Its efficacy results from the medial and inferior walls being attached to large sinuses to which the orbital contents can prolapse after decompression. ${ }^{5}$ However, the eye muscles located at these walls, the medial and inferior rectus muscles, are very often affected most heavily, although it could be shown that in compressive optic neuropathy there is a more generalised impairment of all muscles. ${ }^{6}$ Most often transantral, transconjunctival, transcutaneous, or combined surgical approach is chosen. Many studies substantiate the positive effects of this procedure in patients with compressive orbitopathy. ${ }^{27-10}$

Endoscopic surgical procedures offer an approach to the medial and inferior wall via preformed cavities thus promising a lower incidence of sequelae due to scar formation. Our aim was to analyse a series of data from patients who underwent inferomedial decompression by an endoscopic transnasal approach in order to determine whether this technique is also suitable to sufficiently treat acute orbitopathy with the advantage of avoiding typical Caldwell-Luc complications such as infraorbital pain by scarring and avoiding external cutaneous scars.

\section{VISUAL ACUITY}

Visual acuity could be improved by the procedure in most cases. However, three patients in whom visual acuity was postoperatively diminished in one eye are discussed. In one patient visual acuity decreased from 1.0 to 0.7 in one eye. This was accompanied by a large reduction in abductive capacity from $7.0 \mathrm{~mm}$ to $4.0 \mathrm{~mm}$ and an increase in convergent squint from $7.0^{\circ}$ to $21 \cdot 0^{\circ}$. Following squint operation 5 weeks after operation for decompression, vision returned to $1 \cdot 0$. In another patient vision fell from 0.6 preoperatively to 0.4 at the short term follow up examination. Eight weeks after operation it had returned to $0 \cdot 6$. The third patient preoperatively had a visual acuity of right eye 0.1 and left eye 0.9 . A massive right sided and moderate left sided keratopathy from dry eye syndrome was found during the anterior segment examination not dependent on corneal exposure. Postoperatively, a visual acuity of $0 \cdot 1$ in the right side and 0.5 in the left side was found, probably due to corneal problems. However, 15 months after decompression, which was out of the range of our long term follow up, squint surgery had been performed and vision in the right eye had risen to 0.7 and in the left to $1 \cdot 0$, which shows that postoperative deterioration was not due to a persistent optic nerve problem.

When interpreting data of visual acuity change it should be considered that in our cases bilateral decompression was performed and that clinical experience shows that, generally, one eye is affected more than the other, which is also threatened. This is why mean preoperative visual acuity in group 1 encompassing the more affected eyes rose from 0.35 to 0.59 while in group 2 only a shift from 0.6 to 0.7 could be found, which clinically means no significant change.

It should be noticed that, particularly in those eyes with poor preoperative vision, very good results could be attained. 


\section{PROPTOSIS}

The mean proptosis reduction in our sample was less than reported by Garrity ${ }^{10}$ who, in a large series with transantral decompression, found an average proptosis reduction of $4.7 \mathrm{~mm}$. In his review, Jorissen ${ }^{2}$ also gives proptosis reduction ranging from $4.0 \mathrm{~mm}$ to $6.0 \mathrm{~mm}$ for inferomedial decompression. Mourits, ${ }^{8}$ however, reports a mean proptosis reduction of $2.0 \mathrm{~mm}$ measured by a Hertel exophthalmometer.

In his report on five orbits decompressed by endoscopic approach alone, Kennedy ${ }^{4}$ gives a mean proptosis reduction of $4.7 \mathrm{~mm}$, whereas Mann ${ }^{11}$ found the reduction of the Hertel exophthalmometer median by $1.5 \mathrm{~mm}$ in a collective of 14 patients operated endoscopically.

Remarkably, there was no great difference between groups 1 and 2 as to proptosis. We conclude, from our data, that our technique does not tend to reduce proptosis very much. But it should be stressed that cosmesis was never the indication for surgical intervention in our series. The dissociation between good functional results and only mild proptosis reduction should be marked.

HORIZONTAL SQUINT AND OCULAR MOTILITY

Changes in horizontal squint were accompanied by a marked reduction in abductive capacity, which in the mean was already preoperatively reduced in comparison with normal values. ${ }^{12}$ At the same time adduction was improved.

Postoperative horizontal squint and reduced motility are a problem often mentioned in orbital decompression for any approach. $^{28101113}$ In cases of acute compressive neuropathy probable postdecompressive strabismus or increase of squint angle will not prevent intervention. In our study all patients willing to undergo squint operation for diplopia could be rehabilitated sufficiently.

From our study we conclude that an endoscopic approach ${ }^{411} 14$ for inferomedial decompression in acute compressive orbitopathy can be used sufficiently in order to improve symptoms due to compressive optical neuropathy, especially visual acuity. In cases with disfiguring proptosis additional procedures subsequently or simultaneously might be necessary. An influence on ocular motility stemming from the change of mechanical intraorbital forces due to the effect of the operation cannot be avoided. The long term results show that there is a good protective capacity of the method to avoid recurrence of compressive optic neuropathy. Perioperative complications such as bleeding or dysaesthesia are rare. The endoscopic technique avoids the typical complications of antral approaches and it also avoids disfiguring external scars.

1 Dollinger J. Die Druckentlastung der Augenhöhle durch Entfernung der äußeren Orbitawand bei hochgradigem Exophthalmus und konsekutiver Hornhauterkrankung. Dtsch Med Wochenschr 1911; 37: 1888-90.

2 Jorissen M, Feenstra L. Dysthyroid orbitopathy and orbital decompression - a review. Acta Oto-rhino-laryngol belg 1992; 46: 303-9.

3 Walsh TE, Ogura JH. Transantral orbital decompression for malignant exophthalmos. Laryngoscope 1957; 67: 544-68.

4 Kennedy DW, Goldstein ML, Miller NR, Zinreich SJ Endoscopic transnasal orbital decompression. Arch Otolaryngol Head Neck Surg 1990; 116: 275-82

5 Kulwin DR, Cotton RT, Kersten RC. Combined approach to orbital decompression. Otolaryngol Clin N Am 1990; 23: 381-90.

6 Trobe JD. Optic nerve involvement in dysthyroidism. Ophthalmology 1981; 88: 488-92.

7 Carter KD, Frueh BR, Hessburg TP, Musch DC. Longterm efficacy of orbital decompression for compressive optic neuropathy of Graves' eye disease. Ophthalmology 1991; 98: 1435-42.

8 Mourits MP, Koorneef L, Wiersinga WM, Prummel MF, Berghout A, van der Gaag R. Orbital decompression for Graves' ophthalmopathy by inferomedial plus lateral, and by coronal approach. Ophthalmology 1990; 97: 636-41.

9 Bahn RS, Bartley GB, Gorman CA. Emergency treatment of Graves' ophthalmopathy. Baillière's Clinical Endocrinology and Metabolism 1992; 6: 95-103.

10 Garrity JA, Fatourechi V, Bergstralh EJ, Bartley GB, Beatty CW, DeSanto LW, et al. Results of transantral orbital decompression in 428 patients with severe Graves' ophthalmopathy. Am f Ophthalmol 1993; 116: 533-47.

11 Mann W, Kahaly G, Lieb W, Rothoff T, Springborn S. Orbital decompression for endocrine ophthalmopathy: the endonasal approach. Dev Ophthalmol 1993; 25: 142-50.

12 Mourits MP, Prummel MF, Wiersinga WM, Koornneef L. Measuring eye movements in Graves' ophthalmopathy. Ophthalmology 1994; 101: 1341-6.

13 Fatourechi V, Garrity JA, Bartley GB, Bergstralh EJ, DeSanto LW, Gorman CA. Graves' ophthalmopathy: results of transantral orbital decompression performed primarily for cosmetic indications. Ophthalmology 1994; primarily for

14 Michel O, Bresgen K, Rüssmann W, Thumfahrt WF, Stennert E. Endoskopisch kontrollierte endonasale Orbitadekompression beim malignen ophthalmus. Laryngo-Rhino-Otol 1991; 70: 656-62. 